\title{
29 \\ Trends of Flat Displays in the Multimedia Age
}

\author{
H. Sasaki \\ NEC Corporation \\ 7-1, Shiba 5-Chome, Minato-ku, Tokyo 108-01 Japan \\ Tel:+81-3-3454-1111 Fax:+81-3-3798-6541 \\ e-mail:hajime_sasaki@hq-exec.ccgw.nec.co.jp
}

\begin{abstract}
Today, in a multimedia age, electronic displays are the essential key devices involved in the relation between multimedia equipment and human beings. Flat panel displays are overcoming the restrictions of traditional CRTs (Cathode Ray Tubes) regarding portability and large screen capability, and they are ready to expand their multimedia application fields. TFT-LCDs (Thin Film Transistor-Liquid Crystal Displays) in particular have advantages such as light weight, low profile, low power consumption, and motion picture display, and importantly contribute the development of multimedia. The color PDP (Plasma Display Panel) features emissive display, a large screen, and a low profile, and mass production aimed at implementing space-saving, and wall-hung HDTV (High Definition Television) is about to be started. Since further development of multimedia is expected in the twenty-first century, the scale of this market is immeasurable. At the same time, the flat panel display market will also expand. Wide variety of flat panel displays will gain wider use as they secure their respective markets through their particular advantages.
\end{abstract}

Keywords

Multimedia, Device technology, Electronic display, Flat panel display, LCD, TFT-LCD, Color PDP

\section{Introduction}

The multimedia society has come. Multimedia is not simply a combination of various media. Multimedia contribute to the creation of a sophisticated social life through new forms of 
creation, presentation, understanding, education, and entertainment activities in which the human intellect as well as sensibility are supported by computers.

As multimedia are based on C\&C technologies such as computers, networks, databases, and hypermedia, the development of device technologies including semiconductors, batteries, and electronic displays is the key to implement these technologies.

This article covers the trends of flat panel displays while focusing on TFT-LCDs and color PDPs.

\section{Development of Electronic Devices Supporting Multimedia}

Semiconductor devices have progressed at a remarkable rate for a broad range of applications such as data processing, transmission, and storage. Microprocessors are expected to achieve the remarkably high performance levels ranging from 1000 MIPS to 5000 MIPS. Memory devices are also entering the gigabit generation. The development of ASIC ICs which support the elementary multimedia technologies will directly provide high performance, high functionality and reduced size for multimedia equipment.

The dramatic evolution of semiconductor devices allows image-oriented high-volume data to easily be processed. Much higher levels of multimedia system performance are expected in future.

Electronic displays are essential key devices in the relation between these multimedia equipment and human beings. Among the various electronic displays, traditional CRTs offer general purpose display performance. However, they have limitations regarding portability and large screen. Flat panel displays are overcoming these disadvantages of CRTs, and are about to expand the application field of multimedia. Currently, available flat panel displays are VFDs (Vacuum Fluorescent Displays) in the small size display area, LCDs and EL (Electro-Luminescent) displays in the medium size area, and LED (Light Emitting Diode) array displays and color PDPs in the large size area.

Among these flat panel displays, the evolution of LCDs, in particular of TFT-LCDs, is contributing to the development of multimedia. In addition, color PDPs are ready for full production. Since color PDPs can easily be produced in large screen size, they are expected to grow mainly in the fields of HDTV equipment, space-saving large display, etc.

As secondary batteries having a higher energy density, such as lithium-ion batteries, are increasingly being used as the energy source for the personal and portable equipment, the application fields of flat panel displays will also expand.

\section{Trends of Color TFT-LCDs}

The first LCDs to be introduced were monochrome LCDs for calculators and watches. STN-LCD evolved into large screen sizes, but could not afford high contrast motion picture displays. Higher contrast, higher speed, and full-color flat panel displays were realized by TFT-LCDs which have TFT switches on every pixels. Then, in the early 90's, the large-scale production of color TFT-LCDs started to apply to notebook PCs.

TFT-LCDs can support the wide area of media, since it can display motion pictures as 
well as characters, and still images. LCDs, which offer the advantages of lightweight, low profile, and low power consumption, are increasingly being used for applications linked with multimedia networks. These applications are centered on notebook computers and workstations.

The first generation of TFT-LCDs for notebook computers were for 8 to 9-inch VGA resolution (approx. 0.3 million pixels), while current models are 12-inch SVGA or XGA resolution ( 0.5 to 0.8 million pixels). For $\mathrm{PC}$ monitors and workstations, new products with 13 to 17 -inch screens and SXGA resolution (approx. 1.3 million pixels) are being introduced. Highly finished 20 -inch class models have already been developed.

Mainstream of full-color notebook computer LCDs support 0.26 million colors (with 64level gray scale) by using the multi-bit digital signals. Further progressed full color capability has been achieved with 16.7 million colors (with 256-level gray scale) or with analog signal processing which provides infinite colors similar to CRTs.

The multi-scan resolution expansion technology, which expands the display format from VGA to SXGA, has also been put to practical use. In addition, the adoption of new LCD modes or the development of viewing-angle-expanded-film enables wider viewing angles equivalent to the CRT level.

Portability of a TFT-LCD has been greatly improved. Weight and thickness of TFTLCDs were reduced to approximately half the initial value. Power consumption has decreased to $1 / 4$ the initial level. Reflection type LCDs without a backlight also offer improved display performance. They are expected to come into wide use for portable data terminal applications.

While current TFT-LCDs use a-Si TFTs (amorphous silicon TFTs), higher performance is being realized by shifting to low-temperature-processed $\mathrm{p}$-Si TFTs (polycrystalline silicon TFTs). Low-temperature-processed p-Si TFTs on glass substrates have high electron mobility, and raise TFT-LCD functionality as integration of associated circuits on the substrate glass, opening up the "System on Glass" world.

\section{Trends of Color PDPs}

CRTs or LCDs present manufacturing and practical use restrictions that limit the maximum screen size to approximately 30 - 40 inches. On the other hand, color PDPs offer the same advantages of emissive display and wider viewing angle as CRTs. They also have the advantage that the large flat panel display size more than 40 inches can be produced through a relatively simple manufacturing process.

Comparing with traditional CRTs, the thickness of color PDPs is estimated to be about $1 / 10$, and their weight less than $1 / 5$. In the multimedia age, color PDPs are expected to be the optimal displays for wide-screen flat panel displays, especially for HDTV. In particular, we expect a new market to be created through the implementation of space-saving, large-size wall-hung TVs, something which has been a long-cherished dream.

As the result, the 40 -inch class with $850 \times 480 \times$ RGB pixels, 16.7 million colors, and 200 to $350 \mathrm{~cd} / \mathrm{m}^{2}$ level of luminance has been achieved.

At present, we are pursuing aggressive investment and further improvement for color PDPs as we are preparing for mass production, with the goal of introducing 40 to 60 -inch 
products. To realize a true HDTV, a reduced pixel pitch and faster drive will be important to support an increased number of pixels. The key to meeting these challenges is to obtain higher luminance by improving fluorescent materials and driving method, to optimize manufacturing processes for reduced pixel pitch, and to reduce power consumption.

\section{Future Prospect of Flat Panel Displays}

In the society of twenty-first century, multimedia will grow dramatically and the infrastructure supporting social life will be based in large part on multimedia systems. Along with the development of multimedia, the application of electronic displays will also increasingly expand in the twenty-first century.

Current applications of LCDs focus on personal computers and workstations. In the future, however, the main features of LCDs, i.e. portability, and display performance, will be further improved. Thus, LCDs will further evolve as the indispensable display that can be used anytime, anywhere, and by anybody in the multimedia age, with one display for each person. Also in the monitor market, LCDs will steadily replace CRTs.

Color PDPs, featuring emissive display, large screen size and low profile, will adorn every home as the essential "wall-hung TV". Color PDPs will also steadily grow as they penetrate the public marketplace use.

Wide variety of other flat panel displays will gain wider use as they secure their respective markets through their particular advantages. 